SPICA Workshop, 04013 (2009)

DOI:10.1051/spica/200904013

COwned by the authors, published by EDP Sciences, 2009

This is an Open Access article distributed under the terms of the Creative Commons Attribution-Noncommercial License, which permits unrestricted use, distribution, and reproduction in any noncommercial medium, provided the original work is properly cited.

\title{
WIDE-FIELD STUDY OF DISTANT CLUSTERS OF GALAXIES WITH SPICA
}

\author{
Y. Koyama ${ }^{1}$ and T. Kodama ${ }^{2}$ \\ ${ }^{1}$ Department of Astronomy, School of Science, The University of Tokyo, Tokyo, Japan \\ ${ }^{2}$ National Astronomical Observatory of Japan, Tokyo, Japan
}

\begin{abstract}
We propose an intensive, systematic study of distant clusters of galaxies at $z \sim 0.5-5$ and their surrounding infall regions, taking advantage of the significant wide fieldof-view of MIRACLE $\left(6^{\prime} \times 6^{\prime}\right)$ and the high sensitivity at $\lambda \gtrsim 20 \mu \mathrm{m}$ of MIRACLE and SAFARI. Galaxy clusters and groups are mainly composed of red quiescent galaxies in the local Universe, but the star formation activity becomes much higher in distant clusters. Our coordinated AKARI/Subaru observations have revealed many dusty starburst galaxies in particular in intermediate-density environments such as groups at $z \sim 1$. Interestingly, this is also the same environment in which we see a sharp transition from blue galaxies to red ones. This suggests that dusty starbursts are strongly linked with the physical process that alters the properties of galaxies as they infall and assemble to higher density regions. We also expect that the site of galaxy formation with high star formation activity shifts to higher-density environments as we go back in time, probably due to spatial bias in galaxy formation. By observing many distant clusters at various redshifts between $z \sim 0.5-5$ with SPICA, and identifying dusty, star-forming galaxies across the wide area in and around the clusters/proto-clusters, we will trace back the star formation history of galaxies as a function of environment. We will thus be able to identify directly the epoch and the site of formation of present-day early-type galaxies.
\end{abstract}

Key words: Galaxies: formation - Missions: SPICA

\section{INTRODUCTION}

It is well-established that cosmic star formation rate density is higher in the distant Universe, showing a peak at $z \sim 1-2$. However, since current surveys of the high$z$ Universe are mainly based on the rest-frame UV light, it is always claimed that MIR-FIR study is essential because the UV study may miss a large amount of "hidden" star formation. Therefore, looking back at the distant Universe in MIR-FIR is a fundamental and exciting approach towards understanding the "real" history of the Universe. The situation is even more complicated because properties of galaxies (e.g. morphology, colour, star formation rate) are strongly dependent on environment. For example, in the local Universe, it is well known that high-density regions such as clusters are dominated by red early-type galaxies with little star formation, while most of galaxies in low-density fields are blue, late-type galaxies that are actively forming stars. An important point here is that we need a large sample of galaxies at various redshifts and in various environments to fully understand galaxy evolution and, again, we need MIR/FIR data of them to reveal the hidden side of star formation history.

For this purpose, wide-field MIR observations of distant clusters will be very powerful: galaxy clusters are located in the centre of the large-scale structures at every redshift and we can relatively easily pick up galaxies over a range of different environments such as groups or filaments in the cluster surrounding environment. Taking advantage of the wide-field capability of SPICA, we propose hear an extensive MIR survey of distant galaxy clusters at $0.5 \lesssim z \lesssim 5$, primarily using MIRACLE (and partly SAFARI), to construct large samples of galaxies across a wide range of environments at various redshifts. We should stress here the two great advantages of SPICA, compared with other future IR missions. Firstly, the widefield capability of MIRACLE $\left(6^{\prime} \times 6^{\prime}\right)$ will enable us to map out dusty star formation along distant large-scale structures, even at $z \gg 1$. Secondly, the very high sensitivity at $\lambda \gtrsim 20 \mu \mathrm{m}$ (i.e. MIRACLE at $\lambda \lesssim 40 \mu \mathrm{m}$ and SAFARI at $\lambda \gtrsim 40 \mu \mathrm{m}$ ) will give us a great opportunity to trace dusty star formation in clusters out to $z \sim 5$, based on the rest-frame $7.7 \mu \mathrm{m}$ PAH emission which is an excellent indicator of dusty star formation. In the following sections, dividing into three redshift bins, we describe the possible key science with SPICA that we now strongly support and also mention in passing some of the possible synergies between SPICA and the next generation instruments of Subaru Telescope.

\section{IMPORTANCE OF CLUSTER OUTSKIRTS AT $z \sim 1$}

We have obtained an important motivation for the SPICA cluster survey from our recent wide-field MIR study of a distant cluster RXJ1716.4+6708 at $z=0.81$ with AKARI and Subaru. We found a prominent, large-scale optical structure around the cluster through observations with Suprime-Cam/Subaru (Koyama et al., 2007), and showed that galaxy colours change sharply from blue to red in 

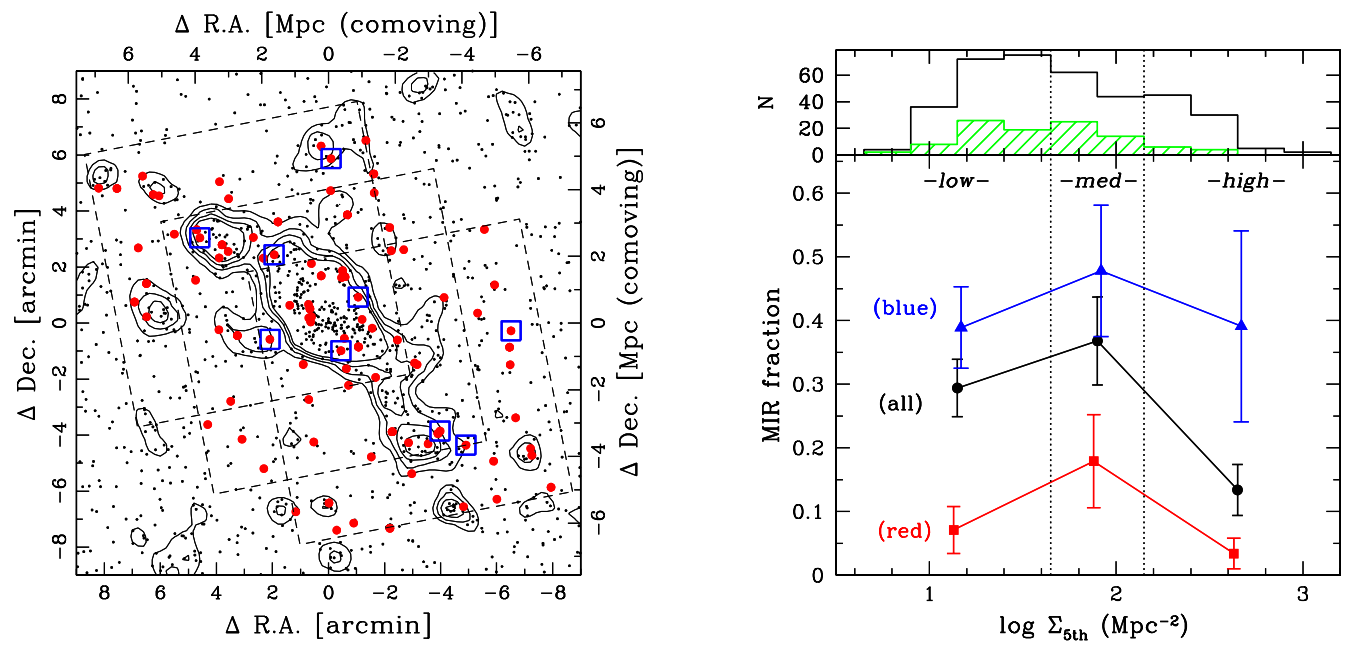

Figure 2. (left): An example of a wide-field mapping of dusty star forming galaxies around a $z \sim 0.8$ cluster, RXJ1716 (Koyama et al., 2008). Small dots show all cluster members, filled circles are MIR-detected galaxies, and open squares are candidates of dusty starbursts which show exceptionally large MIR excess. (right): The MIR fraction in and around the RXJ1716 cluster as a function of local density of galaxies. There is a possible peak of the fraction in the medium-density environment, especially for red galaxies.

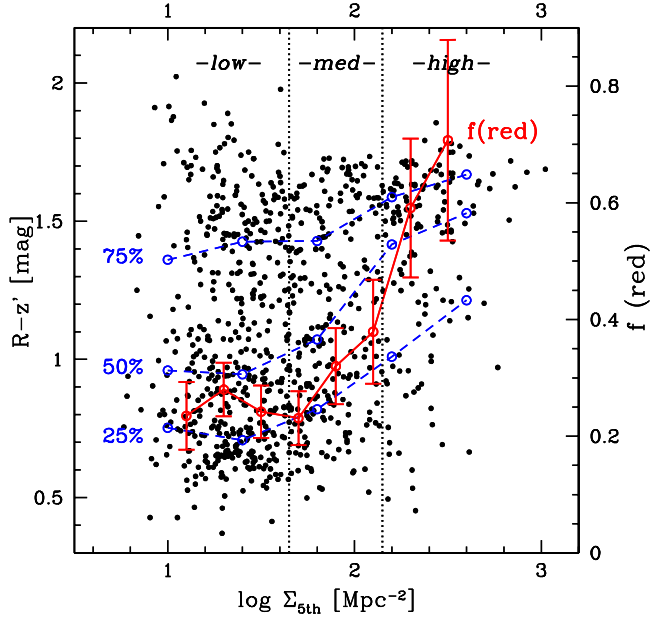

Figure 1. Colour-density plot for the RXJ1716 cluster field. The solid line shows the fraction of red galaxies in each environmental bin, and the vertical dashed-lines define the low-, medium- and high-density environment as indicated.

the "medium-density" environments such as cluster outskirts, groups and filaments (Figure 1, see also Kodama et al., 2001; Tanaka et al., 2005). We note that we cannot know "what happens" in such transition environment from optical information alone, however we have some hints from the MIR observation. We observed in and around the RXJ1716 cluster at $15 \mu \mathrm{m}$ with IRC/AKARI along the large-scale structure (Koyama et al., 2008). This is the first mapping of dusty star formation covering the whole range of environments around a $z \sim 1$ cluster, based on the rest-frame $7.7 \mu \mathrm{m}$ PAH emission. We found many dusty galaxies throughout the structure (Figure 2). Interestingly, we find a possible peak of star forming activity in the "medium-density" environment, which is seen at exactly the same place as the abrupt change of galaxy colours in Figure 1. We also find some optically-red galaxies with MIR detections. This population is relatively rare, but it is interesting to note that $\sim 20 \%$ of red galaxies in the medium-density environment are detected at MIR, while there is almost no such galaxies in high- and low-density environment (Figure 3). Furthermore, we find strong dusty starbursts (identified as a MIR excess galaxies with $\left.z^{\prime}-15 \mu \mathrm{m}>3.7\right)$ are triggered along the large-scale structures around the cluster (open squares in Figure 2). Our recent $\mathrm{H} \alpha$ line survey of this region using a narrowband filter on MOIRCS/Subaru shows that the star formation rates of such very dusty galaxies in the mediumdensity region may be severely underestimated even with $\mathrm{H} \alpha\left(\mathrm{SFR}_{\mathrm{IR}} / \mathrm{SFR}_{\mathrm{H} \alpha} \gtrsim 20\right.$, Koyama et al. 2009.

These findings at $z \sim 0.8$ suggest that the environment where most active star formation occurs may change with redshift, and that in such environments, a major fraction of star formation would be missed by optical star formation indicators because it is hidden by dust. These findings from the wide-field AKARI/Subaru joint survey strongly motivate us to go back to even more distant epochs (see the following sections). At $z \sim 1$, our pioneering survey of cluster surroundings with AKARI/Subaru presented above should be confirmed and extended with SPICA. This is not just an increase of the number of MIR samples of $z \sim 1$ clusters, but we should go deeper and study 

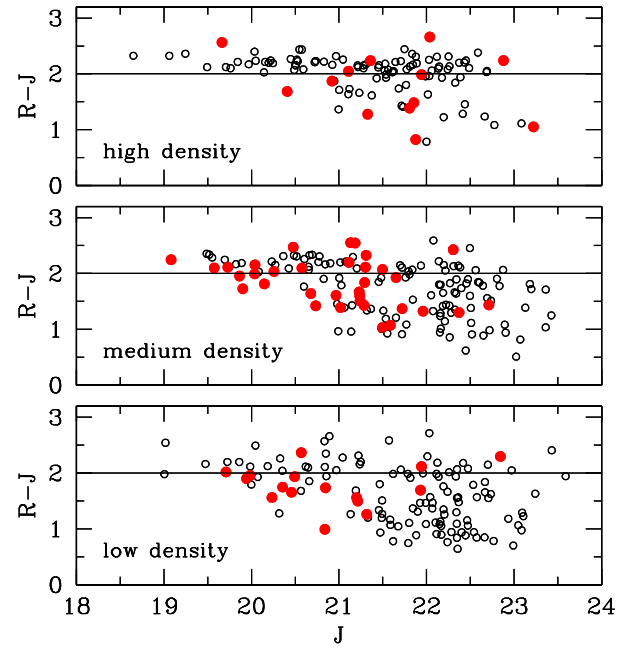

Figure 3. Colour-magnitude diagram for each environment. Note that there are clearly a large number of MIR-detected galaxies (filled circles) on the red-sequence (i.e. dusty red galaxies) in the medium-density region.

the nature of MIR-detected galaxies in $z \sim 1$ clusters in more detail. One of the currently most interesting topics is the challenge to quantify the role of AGNs in the process of cluster formation. It is difficult to distinguish between starbursts and AGNs from a single-band detection at MIR only. However, this problem will be dramatically solved with MIR spectroscopy and/or MIR multi-band photometry (e.g. Takagi et al., 2007), because SEDs of starbursts and AGNs are quite different from each other in the sense that starbursts have prominent PAH emissions while AGNs have power-law SEDs. We will conduct MIR spectroscopic and multi-band MIR photometric surveys, which will enable us to quantify the importance of starbursts/AGNs in distant clusters and their surroundings.

\section{Dusty Star FOrmation In $1 \lesssim z \lesssim 2$ Clusters}

Recent deep and wide-area optical/NIR surveys of clusters at $z>1$ show prominent large-scale structures even around such high- $z$ clusters (e.g. Nakata et al., 2005; Tanaka et al., 2007, 2008). However, the number of known clusters at $1 \lesssim z \lesssim 2$ is still very small, and the star-forming activity in and around such high- $z$ clusters is much less explored compared to $z \lesssim 1$ clusters. Recently, we have an interesting hint that clusters at $z \sim 1.5$ are completely different from $z \lesssim 1$ clusters. We have conducted a deep narrow-band [OII] line survey in a $z=1.45$ cluster, XCS2215 (most distant cluster known to-date), with Suprime-Cam/ Subaru, and find that the fraction of [OII] emitting galaxies is surprisingly high in the cluster central region (Hayashi et al., 2009). This is very different from that of $z \lesssim 1$ clusters where almost no star forming galaxies are found in the cluster core as shown in the previous section. This may suggest that at $z \sim 1.5$ cluster core is still being formed, and galaxy transition (i.e. that seen in cluster outskirts at $z \sim 0.8$ ) is now taking place in the central part of the cluster. If so, it is possible that dusty starbursts coexist in the cluster core, but we couldn't detect them due to the limited number of known clusters at this redshift and the detection limit of the present-day IR telescopes. Thus, we are sure that clusters at $1 \lesssim z \lesssim 2$ are very interesting place to investigate with SPICA, since dusty side of star formation in this redshift range is totally unknown. If we can construct large samples of clusters and largescale structures at $1 \lesssim z \lesssim 2$ in the pre-SPICA era, then a SPICA MIR survey of these young clusters will bring a dramatic improvement in our understanding of galaxy evolution in the early stage of cluster formation. Also, for the study of clusters (and large-scale structures) in this redshift range, we note the importance ande complementarity between the Subaru next generation NIR spectrograph, FMOS, and SPICA. The combination of SPICA and FMOS will allow a super-wide study of $1 \lesssim z \lesssim 2$ clusters along large-scale structures based on MIR and $\mathrm{H} \alpha$, both excellent indicators of dusty and unobscured star formation, respectively.

\section{GOING BACK TO $z \gg 2$ PROTO-CLUSTERS}

We will also target several proto-clusters at $z \gg 2$, which are considered to be in the very early stages of the cluster formation. We will focus on the dusty starbursts and AGNs triggered in such environments because they may be directly related to the formation of cluster early-type galaxies. To-date, mapping the dusty star formation in such high- $z$ clusters at MIR has been almost impossible due to the sensitivity limit of IR telescopes (even with Spitzer and AKARI). However, it is expected that we will be able to detect dusty galaxies up to $z \sim 3$ down to to LIRG level $\left(L_{\mathrm{IR}} \gtrsim 10^{11} L_{\odot}\right)$, through the rest-frame $7.7 \mu \mathrm{m}$ PAH emission with MIRACLE (at $\lambda \sim 30 \mu \mathrm{m}$ ). It is reported that the red-sequence is already apparent in $z \sim 2-3$ proto-clusters, but very massive red galaxies may be seen only at $z \sim 2$ (Kodama et al., 2007). Therefore, it is possible that we can see a formation site of cluster massive galaxies at between $z \sim 2$ and 3 and that such actively forming galaxies are highly dusty. Although the number of known high- $z$ proto-clusters is very limited, we should be able to increase the number prior to the launch of SPICA. We also note that, for $z \gtrsim 2$, the rest-frame $7.7 \mu \mathrm{m}$ PAH emission shifts to $\lambda \gtrsim 20 \mu \mathrm{m}$, where MIRACLE has a higher sensitivity than even the JWST.

Furthermore, we have SAFARI which has a high sensitivity even at $\lambda \gtrsim 40 \mu \mathrm{m}$. Recent optical deep surveys showed possible large-scale structures or clusters up to $z \sim 5-6$ (e.g. Shimasaku et al., 2003; Ouchi et al., 2005), but these over-dense regions are generally traced only by Lyman- $\alpha$ emitters and/or Lyman break galaxies. 
The dusty side of star formation in such regions is totally unknown. Although the FoV of each pointing of SAFARI is smaller than MIRACLE, it will be worth extending our survey out to such high redshift $(z \sim 6)$ with many pointings of SAFARI. Although the sensitivity will be limited to detections of very active galaxies at $z \sim 5-6$ such as ULIRGs, direct detection of dust emission from dusty starbursts in such very high- $z$ clusters will be very exciting, because it has long been believed that large numbers of clusters of early-type galaxies are being formed at these epochs.

\section{Summary}

In summary, we propose a comprehensive survey of distant galaxy clusters at $0.5 \lesssim z \lesssim 5$, through which we will look back on the dusty Universe over a wide range of environments. The wide field and wide wavelength coverage of SPICA (especially MIRACLE and SAFARI) will enable us to do this based on the identical tracer (i.e. $7.7 \mu \mathrm{m}$ PAH emissions) of dusty star formation, and also to unveil the importance of hidden activities of galaxies along the cluster formation history. Through this project, SPICA can effectively construct large samples of galaxies at various redshifts and various environments, which will allow us to add the environmental axis to the plot of cosmic star formation history (Figure 4). This is clearly an important step towards gaining a complete understanding of the history of the Universe, and at the same time we will identify the "most active" environments as a function of redshift. As suggested in Figure 4, star formation may be higher in clusters than low-density fields at $z \gg 1$, however this will only be clear once we have a MIR view of the highdensity region in clusters at such high redshift Although the number of known clusters at $z \gg 1$ is still very limited, it should be possible to construct much larger samples of $z \gg 1$ clusters in the pre-SPICA era, thus preparing for the SPICA launch.

\section{ACKNOWLEDGEMENTS}

The MIR part of the result of the RXJ1716 cluster presented here is based on observation with AKARI, a JAXA project with the participation of ESA. The optical/NIR data is collected with Subaru, which is operated by National Astronomical Observatory of Japan. Y.K. acknowledge support from the Japan Society for the Promotion of Science (JSPS) through JSPS fellowships for young scientists.

\section{REFERENCES}

Hayashi, M., et al. 2009, MNRAS, submitted Kodama, T., et al. 2007, MNRAS, 377, 1717

Kodama, T., et al. 2001, ApJL, 562, L9

Koyama, Y., et al. 2009, in prep.

Koyama, Y., et al. 2008, MNRAS, 391, 1758

Koyama, Y., et al. 2007, MNRAS, 382, 1719

Nakata, F., et al. 2005, MNRAS, 357, 1357

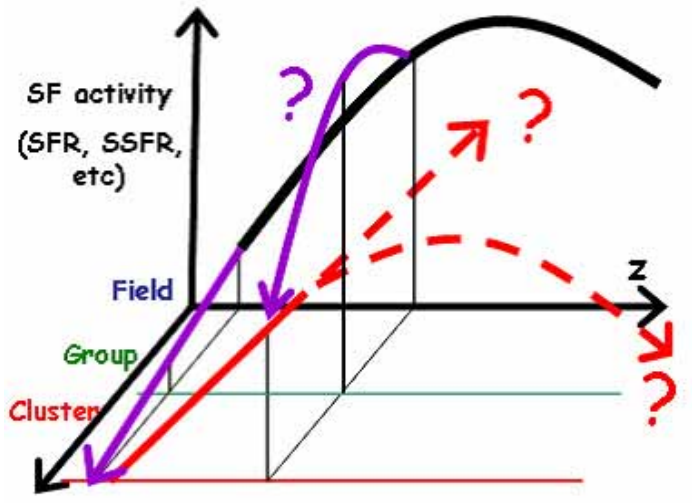

Figure 4. A summary plot of our planning project. We will reveal the cosmic star formation history not only through the blank-field survey but through the survey of wide range of environment, so that we can add an environmental axis in the plot of cosmic star formation history.

Ouchi, M., et al. 2005, ApJL, 620, L1

Shimasaku, K., et al. 2003, ApJL, 586, L111

Takagi, T., et al. 2007, PASJ, 59, 557

Tanaka, M., et al. 2008, A\&A, 489, 571

Tanaka, M., et al. 2007, MNRAS, 377, 1206

Tanaka, M., et al. 2005, MNRAS, 362, 268 